http://dx.doi.org/ 10.22319/rmcp.v9i4.4333

Artículo

Uso de retenedores de humedad edáfica en la sobrevivencia y crecimiento de dos especies de pastos Bouteloua curtipendula [Michx.] Torr. y Chloris gayana Kunth

Use of soil moisture retainers on the survival and growing of two grass species Bouteloua curtipendula [Michx.] Torr. and Chloris gayana Kunth

Luis Gerardo Yáñez-Chávez

Aurelio Pedroza-Sandoval ${ }^{\mathrm{a}^{*}}$

Martín Martínez-Salvador ${ }^{\mathrm{b}}$

Ignacio Sánchez-Cohen ${ }^{\mathrm{c}}$

Francisco Guadalupe Echavarría-Cháirez ${ }^{\mathrm{d}}$

Miguel Agustín Velásquez-Valle

Armando López-Santos ${ }^{\mathrm{a}}$

${ }^{a}$ Universidad Autónoma Chapingo. Unidad Regional Universitaria de Zonas Áridas. Km 40 Carretera Torreón-Chihuahua. 35230 Bermejillo, Durango. México.

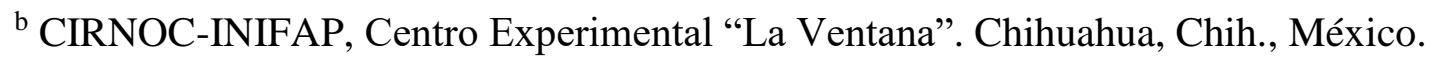

${ }^{\mathrm{c}}$ INIFAP, Centro Nacional de Investigación Disciplinaria en Relaciones Agua Suelo Planta Atmósfera, Gómez Palacio, Durango. México.

${ }^{\mathrm{d}}$ CIRNOC-INIFAP. Centro Experimental de Calera, Calera, Zacatecas, México.

${ }^{\text {e }}$ CIRNE-INIFAP. Campo Experimental Saltillo, Saltillo, Coahuila, México.

*Autor de correspondencia: apedroza@ chapingo.uruza.edu.mx 


\section{- Resumen:}

El $85 \%$ de las zonas áridas y semiáridas de México presentan problemas de erosión por sobrepastoreo en áreas de pastizal, con el consecuente incremento en el proceso de desertificación de los suelos. El objetivo del presente estudio, fue evaluar diferentes especies y prácticas de retención de humedad en el suelo en el establecimiento y desarrollo de pasto en zonas semiáridas. Se usó un diseño experimental de bloques al azar en un arreglo de parcelas divididas con tres repeticiones. Las parcelas grandes correspondieron a las dosis de rastrojo de maíz (0 y $\left.10 \mathrm{t} \mathrm{ha}^{-1}\right)$, las parcelas medianas a las dosis de hidrogel $(0,10$ y $20 \mathrm{~kg}$ $\mathrm{ha}^{-1}$ ) y las parcelas chicas a las dos especies de pastos Bouteloua curtipendula [Michx.] Torr. y Chloris gayana Kunth. Se usó el método de trasplante. El porcentaje de sobrevivencia fue superior a $72 \%$ en promedio general, aun cuando B. curtipendula fue sobresaliente en las diferentes fechas de evaluación. El rastrojo de maíz como cobertura vegetal, registró un contenido de humedad en el suelo de $16.9 \%$ en relación al testigo, con el consecuente aumento de $24.9 \%$ en la producción de biomasa en términos de materia seca. Lo anterior se asoció a plantas con mayor altura, un mayor número de macollos y un mayor contenido de clorofila. El hidrogel, sólo influyó durante los primeros 15 días después del trasplante al inicio de elongación, con un mejor porcentaje de sobrevivencia después del trasplante de los pastos; sin ningún efecto en fechas posteriores durante la fase de crecimiento y desarrollo del pasto.

- Palabras clave: Cobertura vegetal, Rastrojo de maíz, Hidrogel, Forraje, Pastizal.

\section{- Abstract:}

About $85 \%$ of the arid and semiarid zones of Mexico have erosion problems related to overgrazing in livestock farms, with the consequent increase in the degradation of the soil. The objective of the study was to evaluate different species and practices of soil moisture retention in the survival and development of grass in semiarid lands. A random experimental block design was used in a split-split plots arrangement with three replications. The large plots corresponded to the doses of stubble of corn $\left(0\right.$ and $\left.10 \mathrm{t} \mathrm{ha}^{-1}\right)$, medium plots were the hydrogel doses $\left(0,10\right.$ and $\left.20 \mathrm{~kg} \mathrm{ha}^{-1}\right)$ and small plots were the two species of grass Bouteloua curtipendula [Michx.] Torr, and Chloris gayana Kunth. The transplanting method was used. The survival percentage was greater than $72 \%$ from general average; but this variable was outstanding in the $C$. curtipendula during different evaluation dates. The corn stubble as crop coverture increased the soil moisture content in $16.9 \%$ in relation to the control, it as consequent increase of $24.9 \%$ in biomass production in terms of dry matter. Before it was associated with plants of higher height, and higher number of tillers and higher chlorophyll content. The hydrogel in any doses used, only influenced the better of survival percentage of the grasses at the first $15 \mathrm{~d}$ after transplanting, when started the elongation step, but not in their growing and development. 
- Key words: Crop coverture, Dry corn stubble, Hydrogel, Forage, Pasture.

Recibido 30/11/2016

Aceptado 22/01/2018

\section{U Introducción N}

Las características más recurrentes en los pastizales de las zonas áridas y semiáridas son las sequías y la alta presión del uso del suelo por el sobrepastoreo ${ }^{(1)}$. Los sistemas poco productivos en las regiones áridas y semiáridas con escasa cobertura vegetal en el suelo y bajo contenido de materia orgánica, son más proclives al proceso de erosión y desertificación ${ }^{(2,3,4)}$.

La degradación de los recursos naturales de los pastizales en las zonas áridas y semiáridas como una forma de desertificación, es lo que más afecta al ecosistema donde se practica ganadería extensiva $^{(2)}$. El manejo inadecuado de las áreas de pastoreo, ha propiciado una degradación física, lo cual las hace más vulnerables a la errática precipitación en la temporada de lluvia y a precipitaciones menores a las esperadas, generándose un problema para los habitantes de las comunidades rurales por la disminución de la capacidad productiva de sus áreas de agostadero $^{(5)}$. Para minimizar los efectos de las sequías en las zonas áridas y semiáridas, es importante incorporar prácticas que permitan mitigar los efectos de años secos y su impacto en la economía de las comunidades de estas regiones ${ }^{(1)}$. El establecimiento de pastos en suelos con degradación física, implica la posibilidad de obtener alimento para el ganado y mejorar la condición del terreno.

La cuenca media de los ríos Nazas-Aguanaval, es un área predominantemente de pastizal en condición árida. Esta región registra un deterioro ambiental por la existencia de terrenos dedicados a la ganadería extensiva con sobrepastoreo y la consecuente pérdida parcial de la cubierta vegetal y de suelo ${ }^{(6)}$. Para la rehabilitación de esta zona, es importante considerar especies de pastos tolerantes a las condiciones marginales y de estrés, tanto de tipo biótico, como abiótico $^{(7)}$. La incorporación al suelo de coberturas vegetales, así como el uso de retenedores de humedad, representan tecnologías para reducir la alta tasa de evaporación y prolongar la disponibilidad del agua para las plantas. Este tipo de tecnologías son aún poco 
exploradas y no bien valoradas para un eficiente establecimiento de vegetación en suelos degradados por efecto de la erosión y desertificación en zonas áridas. El objetivo de este estudio fue evaluar diferentes prácticas de retención de humedad en el suelo para el establecimiento y desarrollo de pastos en áreas degradadas de zonas áridas.

\section{Material y métodos V}

El estudio se llevó a cabo en San Luis del Cordero, Durango, México en 2015. La región se encuentra ubicada en los paralelos $25^{\circ} 15^{\prime}$ y $25^{\circ} 31^{\prime} \mathrm{N}$ y los meridianos $104^{\circ} 07^{\prime}$ y $104^{\circ} 33^{\prime}$ $\mathrm{O}$, a una altitud de $1,508 \mathrm{~m}$. La temperatura máxima es de $40^{\circ} \mathrm{C}$ en los meses de mayo a agosto, y la mínima es de $0{ }^{\circ} \mathrm{C}$ entre diciembre y marzo. El régimen de precipitación es de $362.1 \mathrm{~mm}$ anuales y ocurre de junio a octubre ${ }^{(8)}$.

\section{- Diseño experimental}

Se usó un diseño experimental de bloques al azar en un arreglo de parcelas divididas con tres repeticiones. Las parcelas grandes fueron las dosis de rastrojo de maíz ( 0 y $\left.10 \mathrm{t} \mathrm{ha}^{-1}\right)$; las parcelas medianas correspondieron a las dosis de hidrogel (0, 10 y $\left.20 \mathrm{~kg} \mathrm{ha}^{-1}\right)$; y las parcelas chicas fueron las dos especies de pastos B. curtipendula [Michx.] Torr.) y C. gayana Kunth (el primero corresponde a un pasto nativo y el segundo a un pasto introducido). Cada unidad experimental fue de 12 surcos de $12 \mathrm{~m}$ de largo x $5 \mathrm{~m}$ de ancho, con una superficie experimental de $2,160 \mathrm{~m}^{2}$. La parcela útil correspondió a los seis surcos centrales, en los cuales se seleccionaron cuatro plantas al azar en las que se midieron las variables correspondientes.

La siembra del pasto se realizó en charolas de unicel en condiciones de invernadero el 15 de agosto de 2015. Como sustrato se utilizó "peat moss". Se aplicaron riegos ligeros uniformes diariamente, evitando la deshidratación de las plántulas. La germinación ocurrió a los tres días después de la siembra en las dos especies de pasto. 
El trasplante se hizo manualmente en el área experimental el 19 de septiembre de 2015 . El rastrojo de maíz se aplicó superficialmente como cobertura vegetal antes del trasplante de los pastos. La aplicación del hidrogel se realizó manualmente, al mismo tiempo que el trasplante. El hidrogel se depositó a la misma profundidad que el cepellón de la plántula, aplicando 1 y $2 \mathrm{~g}$ planta $^{-1}$. La distancia entre plantas fue $50 \mathrm{~cm}$, con una densidad de 26,667 plantas ha ${ }^{-1}$. Los hidrogeles son copolímeros granulados que tienen un contenido de materia seca de 85 a $90 \%$, densidad aparente de $0.85 \mathrm{~g} \mathrm{ml}^{-1}$, peso específico de $1.10 \mathrm{~g} \mathrm{~cm}^{-3} \mathrm{y} \mathrm{pH}$ de $8.1^{(9)}$; tienen una capacidad de incrementar la retención de humedad del suelo y con ello aprovechar mejor el agua de lluvia o riego.

El temporal de lluvias ocurre en los meses de junio a octubre, donde la precipitación es suficiente para el desarrollo de los cultivos en la región ${ }^{(8)}$. Un escurrimiento superficial previo al trasplante, producto de un lluvia ocurrida el 11 de septiembre del 2015, con una precipitación de $27.8 \mathrm{~mm}$, permitió el trasplante. La precipitación acumulada fue de $40.4 \mathrm{~mm}$ in situ entre los meses de septiembre a noviembre.

\section{- Variables}

Durante la etapa de germinación y crecimiento de la plántula en las charolas se midió la velocidad de germinación de acuerdo a la ecuación ${ }^{(10)}$ :

$$
\mathbf{V G}=\sum \frac{(\mathbf{n i})}{\mathbf{t}}
$$

\section{Donde:}

$\mathbf{V G}=$ velocidad de germinación;

$\mathbf{n i}=$ número de semillas germinadas en el día;

$\mathbf{T}=$ tiempo de germinación desde la siembra hasta la germinación de la última semilla.

En la etapa de campo, a los 15, 30, 45 y 60 días después del escurrimiento (DDE), se midió: contenido de humedad en el suelo (\%) a las profundidades de 15 y $30 \mathrm{~cm}$, mediante un determinador de humedad Lutron Modelo PMS-714 (Taipei, TPE); sobrevivencia de plántula (\%), con un sistema de muestreo aleatorio con tramos de $3 \mathrm{~m}$ lineales por tratamiento a los 15 DDE; número de macollos, mediante conteo directo por planta; altura de planta $(\mathrm{cm})$, con uso de cinta métrica graduada; índice de clorofila (ICC), con medidor de clorofila Fieldscout 
CM 1000 (Plainfield IL, USA); contenido de materia seca (g) de la parte aérea y la raíz de la planta, mediante muestreo destructivo y secado en estufa de aire recirculante, HAFO® modelo 1600 , USA, a $75^{\circ} \mathrm{C}$ por $36 \mathrm{~h}$.

\section{- Análisis estadístico}

Se realizaron análisis de varianza y prueba de medias de Tukey $(P \leq 0.05)$ entre tratamientos, con sus respectivas interacciones entre factores de variación en cada variable, así como un análisis de regresión para obtener las tasas de crecimiento con los modelos de mejor ajuste en la velocidad de germinación de semillas. Se usó el paquete estadístico SAS® Versión $9.0^{(11)}$.

\section{\| Resultados y discusión \|}

Por la ausencia de interacción entre las especies de pastos con la cobertura vegetal y dosis de hidrogel, se procedió a la exposición de los resultados del crecimiento y desarrollo de las dos especies de pasto de manera independiente a los factores de variación citados.

\section{- Velocidad de germinación •}

La tasa logarítmica de germinación de $B$. curtipendula fue significativamente mayor $(P \leq 0.05)$ respecto a $C$. gayana, con valores de $106.2\left(\mathrm{R}^{2}=0.97\right)$ y $91.9\left(\mathrm{R}^{2}=0.95\right)$, respectivamente. (Figura 1). Este efecto de respuesta está relacionado al mecanismo de latencia de las especies nativas, referido a una alta capacidad de germinación ${ }^{(12)}$; pero aun cuando se tenga un porcentaje alto de germinación en especies exóticas, no existen evidencias 
claras para su éxito en la producción potencial ${ }^{(12)}$. Adicionalmente, se indica que una característica del pasto banderita (C. curtipendula), es su rápida velocidad de germinación ${ }^{(13)}$.

Figura 1: Tasa de germinación (no. de semillas día ${ }^{-1}$ ) en pastos B. curtipendula [Michx.] Torr. y C. gayana Kunth

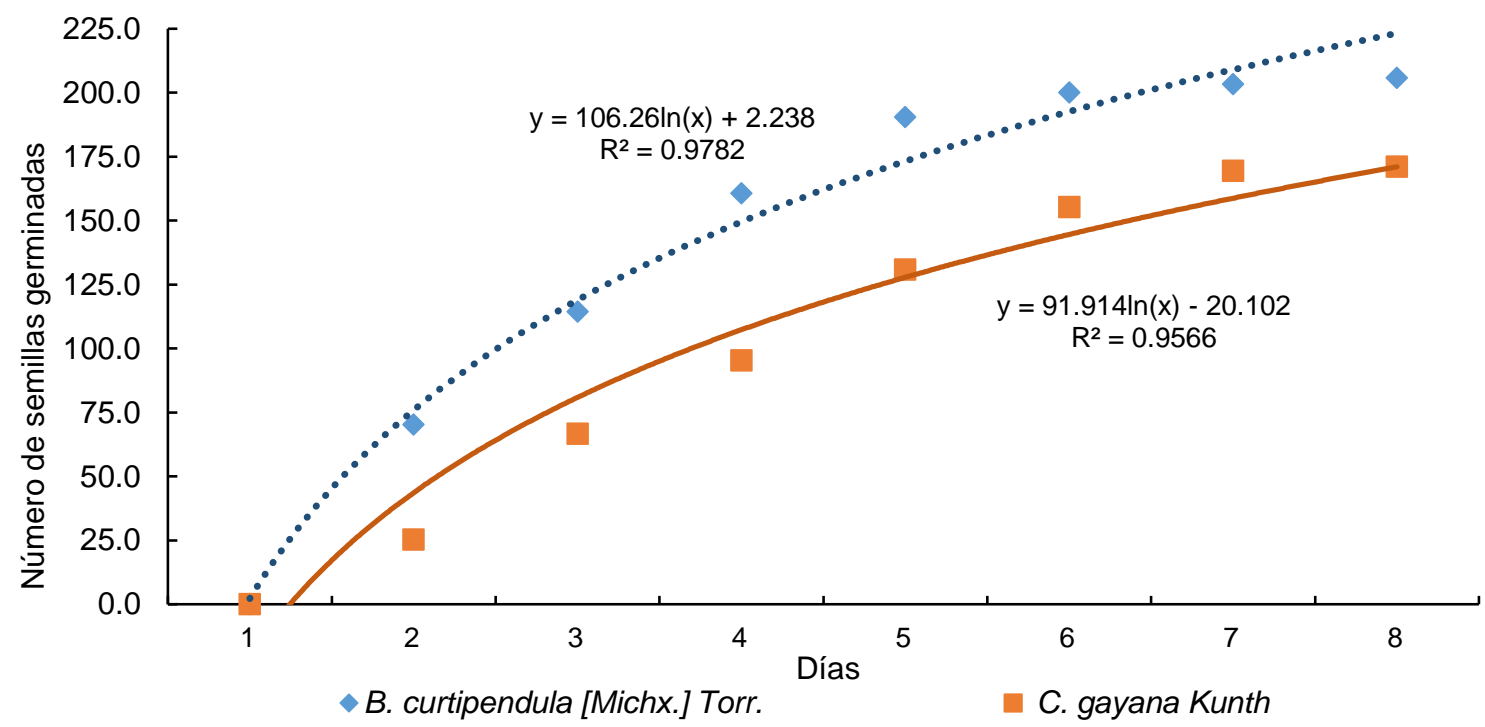

- Contenido de humedad en el suelo •

El contenido de humedad fue significativamente $(P \leq 0.05)$ mayor al aplicar hidrogel a los 15 días después del escurrimiento (DDE) en cada profundidad evaluada, al registrar valores en promedio $3 \%$ superiores, con respecto al testigo; este efecto se disipó en las fechas posteriores de evaluación para ambas profundidades. La tasa exponencial de abatimiento de humedad en el tiempo a los $15 \mathrm{~cm}$ de profundidad fue de $-0.16\left(R^{2}=0.96\right),-0.14\left(R^{2}=0.98\right)$ y $-0.10\left(R^{2}=0.98\right)$ en los tratamientos de 20,10 y $0 \mathrm{~kg} \mathrm{ha}^{-1}$ de hidrogel, respectivamente. Un comportamiento similar se registró a los $30 \mathrm{~cm}$ de profundidad, con tasas de $-0.09 \quad\left(\mathrm{R}^{2}=\right.$ $0.99),-0.13\left(R^{2}=0.97\right)$ y $-0.13\left(R^{2}=0.95\right)$ en los tratamiento de 20,10 y $0 \mathrm{~kg} \mathrm{ha}^{-1}$ de hidrogel, respectivamente (Figura 2). El hidrogel posee propiedades de retención y liberación lenta de agua en el suelo, ya sea en condiciones de irrigación instantánea o prolongada, además de conservar la humedad en la rizósfera de los cultivos ${ }^{(14)}$; sin embargo en este estudio, las propiedades del hidrogel no se observaron en los muestreos posteriores a los 15 días después 
del escurrimiento registrado. Estos resultados no coinciden con lo reportado por otros investigadores, quienes encontraron un mayor contenido de humedad del suelo al aplicar hidrogel en plantas de Zea mays, lo cual incrementó significativamente la biomasa y optimizó el uso de agua por el cultivo ${ }^{(15)}$. Otros autores destacan que las aplicaciones de hidrogel en pasto buffel en climas áridos, mejoraron la emergencia de plántula, altura de planta, peso de materia seca y cobertura vegetal ${ }^{(7)}$, lo cual puede estar asociado a que los hidrogeles mejoran significativamente la capacidad de absorción de agua fácilmente extraíble (RAW, por sus siglas en inglés) de los suelos; aunque la eficacia del gel en mejorar la retención de agua, varía de acuerdo al tipo de suelo ${ }^{(16)}$.

Figura 2: Abatimiento de humedad a $15 \mathrm{~cm}$ (A) y $30 \mathrm{~cm}$ (B) de profundidad del suelo con diferentes contenidos de hidrogel y diferentes fechas de muestreo
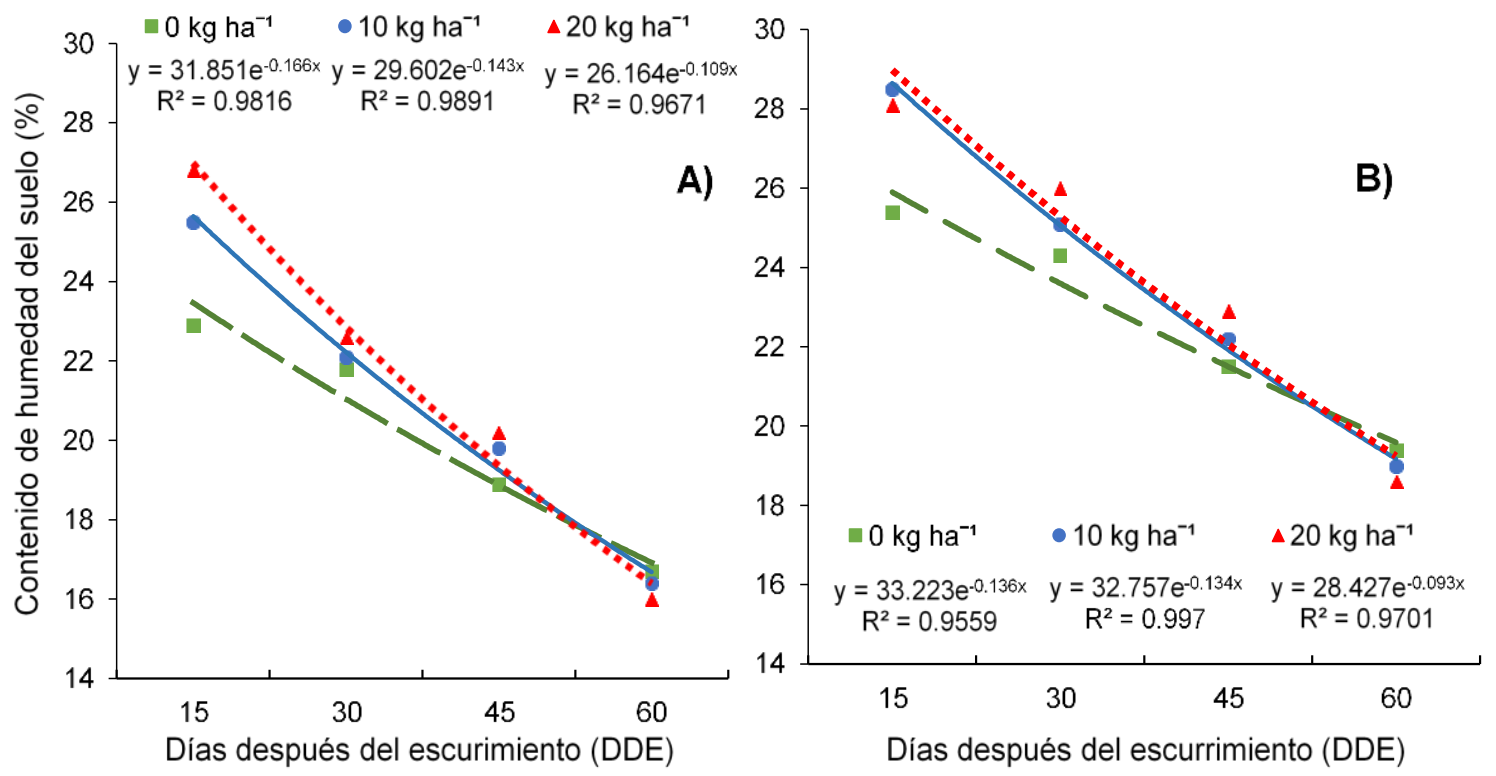

La capacidad del hidrogel de retener el agua en el suelo, se asocia a la constante hidratación, condición que no se presentó en el sitio de estudio, debido a las bajas precipitaciones registradas durante el año. La ocurrencia de precipitaciones para zonas áridas y semiáridas es poco homogénea y variable en el tiempo.

Respecto al uso de cobertura vegetal a base de rastrojo de maíz, el contenido de humedad en ambas profundidades del suelo, se mantuvo superior $(P \leq 0.05)$ en $3.2 \%$ en cada fecha de evaluación con respecto a donde no se adicionó el residuo de cosecha (Figura 3). La humedad aprovechable para este tipo de suelo es $18 \%$, dado que la CC es $33 \%$ y el PMP de $15 \%$. El contenido de humedad edáfica registró valores de $16 \%$ en el tratamiento sin aplicación de 
rastrojo a 45 y 60 DDE, muy próximos al PMP; en cambio, donde se aplicó el rastrojo, el suelo siempre mantuvo contenidos de humedad superiores a $18.0 \%$, con una tasa de abatimiento de humedad exponencial negativa de $-0.12\left(\mathrm{R}^{2}=0.99\right)$ y $-0.18\left(\mathrm{R}^{2}=0.98\right)$ en la profundidad de $15 \mathrm{~cm}$ y $30 \mathrm{~cm}$, respectivamente. En tanto que, el tratamiento sin rastrojo registró tasas de $-0.13\left(\mathrm{R}^{2}=0.99\right)$ y $-0.14\left(\mathrm{R}^{2}=0.97\right)$ a $\operatorname{los} 15$ y $30 \mathrm{~cm}$ de profundidad, respectivamente. En promedio, el tratamiento con rastrojo de maíz registró 3.7 y $3.1 \%$ más contenido de humedad, con respecto al testigo a los 15 y $30 \mathrm{~cm}$ de profundidad, respectivamente.

Figura 3: Abatimiento de humedad a $15 \mathrm{~cm}$ (A) y $30 \mathrm{~cm}$ (B) de profundidad del suelo con y sin cobertura a base de rastrojo de maíz en diferentes fechas de muestreo
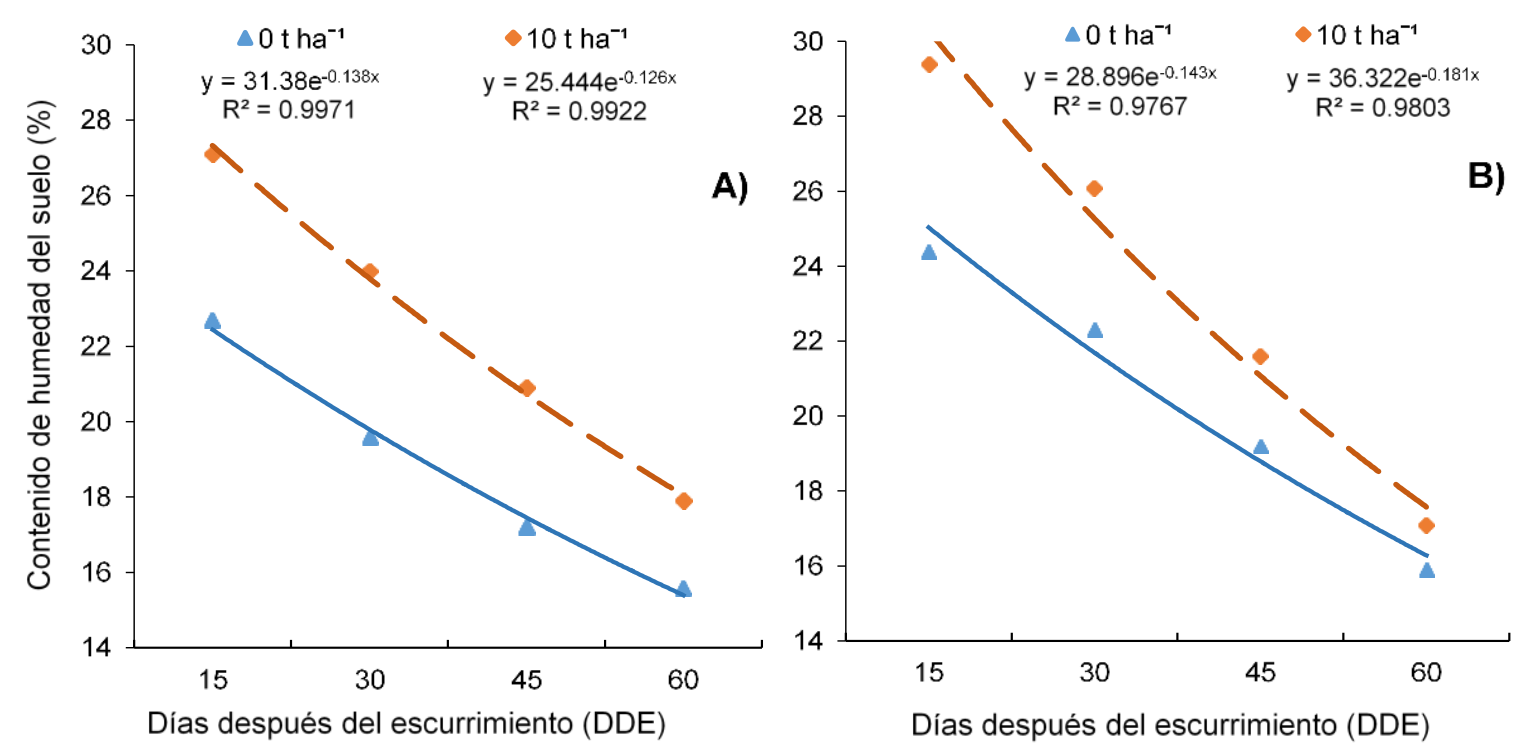

Los anteriores resultados coinciden con estudios que usaron mantillo o residuo de cosecha en la producción de soya y sorgo de secano, y obtuvieron un mayor contenido de humedad en el suelo al aplicar mantillo en ambos cultivos, principalmente en años con precipitaciones irregulares $^{(17)}$. Adicionalmente, se indica que la incorporación de mantillo o rastrojos a los cultivos, representa una práctica cultural importante, ya que desempeña un papel esencial en la conservación de humedad en el suelo; las coberturas orgánicas e inorgánicas, en promedio registraron un contenido más alto de humedad edáfica para la capa activa del suelo $(0-40 \mathrm{~cm})$ en tres años de evaluación, con un $18 \%$ de humedad en campo con cobertura de paja, mientras que el tratamiento sin cobertura obtuvo $16.2 \%$ de humedad ${ }^{(18)}$. La aplicación combinada de paja y mantillo e incorporación de paja al primer perfil de suelo, mejoró la retención de agua del suelo en 2.1 a $10.4 \%$ y la salinidad de la capa superficial disminuyó 5.4 a $23.0 \%{ }^{(19)}$. 


\section{- Sobrevivencia de plántula}

B. curtipendula y C. gayana tuvieron una supervivencia superior al $84 \%$, seis semanas después del trasplante, sin diferencias estadísticas entre ambas especies. El alto porcentaje de sobrevivencia, pudiera estar asociados al método de siembra en trasplante, ya que el método simple de siembra directa de semilla en campo, es sólo del $10 \%$ y en algunos casos $50 \%{ }^{(10)}$. Se han encontrado valores superiores al $95 \%$ de establecimiento en pasto buffel al utilizar el método del trasplante, por lo que es considerado un método de siembra altamente efectivo aún en suelos con limitada fertilidad natural ${ }^{(10)}$.

El porcentaje de sobrevivencia de los pastos fue de 89.3 y $76.4 \%$ al aplicar $20 \mathrm{~kg} \mathrm{ha}^{-1}$ y 10 $\mathrm{kg} \mathrm{ha}^{-1}$, respectivamente, sin diferencia estadística entre ambos, con una mejor tendencia de respuesta en la dosis de $20 \mathrm{~kg} \mathrm{ha}^{-1}$, al diferenciarse estadísticamente del testigo. El porcentaje de sobrevivencia fue mayor $(P<0.05)$ al aplicar el rastrojo de maíz $(89.9 \%)$, respecto cuando no se aplicó $(81.2 \%)$ (Cuadro 1). Al no aplicar la cobertura vegetal, el porcentaje de sobrevivencia de especies forestales se reduce significativamente, hasta un $66.7 \%{ }^{(20)}$. La aplicación de rastrojo como cobertura vegetal en los cultivos, mejora la retención de humedad del suelo al reducir la evaporación, además de crear un microclima (temperatura y humedad) adecuado para la germinación de la semilla, supervivencia y desarrollo del cultivo en su fase inicial ${ }^{(21)}$.

Cuadro 1: Porcentaje de sobrevivencia por especie de pasto, diferentes dosis de hidrogel y cobertura vegetal a base de rastrojo de maíz

\begin{tabular}{lc}
\hline $\begin{array}{l}\text { Especie de pasto/dosis de retenedor de } \\
\text { humedad }\end{array}$ & Porcentaje de sobrevivencia \\
\hline B. curtipendula $[$ Michx.] Torr & $87.03 \mathrm{a} \pm 1.3$ \\
C. gayana $\left.\mathrm{Kunth}^{-1}\right)$ : & $84.12 \mathrm{~b} \pm 1.1$ \\
Hidrogel $\left(\mathrm{kg} \mathrm{ha}^{-1}\right)$ & $72.93 \mathrm{~b} \pm 0.9$ \\
0 & $76.42 \mathrm{~b} \pm 1.5$ \\
10 & $88.64 \mathrm{a} \pm 1.2$ \\
20 & \\
Rastrojo de maíz $\left(\mathrm{t} \mathrm{ha}^{-1}\right):$ & $81.21 \mathrm{~b} \pm 1.4$ \\
0 & $89.94 \mathrm{a} \pm 1.6$ \\
10 & $(P<0.05)$.
\end{tabular}




\section{- Número de macollos, altura de planta e índice de clorofila •}

No hubo efecto de interacción entre la cobertura con rastrojo de maíz y las dosis de hidrogel utilizadas en ninguna de las características de desarrollo y crecimiento de la planta. En un análisis estadístico por factor de variación por separado, tampoco el hidrogel mostró efecto en ninguna de las variables antes citadas. La cobertura vegetal a base de rastrojo de maíz a dosis de $10 \mathrm{t} \mathrm{ha}^{-1}$ mejoró significativamente $(P \leq 0.05)$ todas las variables: la altura de planta registró $37.2 \mathrm{~cm}, 3.8$ macollos e índice de clorofila con 121.5 en el pasto introducido; el pasto nativo reportó valores de altura de $31.9 \mathrm{~cm}, 7.1$ macollos e índice de clorofila de 98.5. El pasto introducido fue superior $55.5 \%$ en altura, $16.6 \%$ en macollos y $23.3 \%$ en el índice de clorofila con respecto al pasto nativo (Cuadro 2).

Cuadro 2: Características morfométricas en dos especies de pasto en diferentes dosis de hidrogel y cobertura vegetal a base de rastrojo de maíz

\begin{tabular}{|c|c|c|c|c|c|c|}
\hline \multirow{2}{*}{$\begin{array}{l}\text { Dosis de retenedor } \\
\text { de humedad }\end{array}$} & \multicolumn{2}{|c|}{ Número de macollos } & \multicolumn{2}{|c|}{ Altura de planta $(\mathrm{cm})$} & \multicolumn{2}{|c|}{ ICC } \\
\hline & $\mathbf{B C}$ & CG & $\mathbf{B C}$ & CG & $\mathbf{B C}$ & CG \\
\hline \multicolumn{7}{|c|}{ Hidrogel $\left(\mathrm{kg} \mathrm{ha}^{-1}\right)$} \\
\hline 0 & $\begin{array}{l}5.6^{\mathrm{a}} \\
\pm 1.0\end{array}$ & $\begin{array}{l}3.5^{\mathrm{a}} \\
\pm 0.8\end{array}$ & $\begin{array}{l}26.9^{\mathrm{a}} \\
\pm 4.8\end{array}$ & $\begin{array}{l}33.1^{\mathrm{a}} \\
\pm 4.8\end{array}$ & $\begin{array}{l}98.6^{\mathrm{a}} \\
\pm 5.3\end{array}$ & $\begin{array}{r}114.3^{\mathrm{a}} \\
\pm 5.1\end{array}$ \\
\hline 10 & $\begin{array}{l}5.7^{\mathrm{a}} \\
\pm 0.9\end{array}$ & $\begin{array}{l}3.6^{\mathrm{a}} \\
\pm 0.9\end{array}$ & $\begin{array}{l}27.7^{\mathrm{a}} \\
\pm 5.2\end{array}$ & $\begin{array}{l}34.9^{\mathrm{a}} \\
\pm 4.6\end{array}$ & $\begin{array}{l}95.9^{\mathrm{a}} \\
\pm 4.7\end{array}$ & $\begin{array}{c}113.2^{\mathrm{a}} \\
\pm 5.7\end{array}$ \\
\hline 20 & $\begin{array}{l}5.9^{\mathrm{a}} \\
\pm 1.1\end{array}$ & $\begin{array}{l}3.2^{\mathrm{a}} \\
\pm 0.9\end{array}$ & $\begin{array}{l}27.2^{\mathrm{a}} \\
\pm 4.3\end{array}$ & $\begin{array}{l}32.6^{\mathrm{a}} \\
\pm 3.6\end{array}$ & $\begin{array}{l}97.8^{\mathrm{a}} \\
\pm 4.3\end{array}$ & $\begin{array}{r}109.8^{\mathrm{a}} \\
\pm 6.2\end{array}$ \\
\hline \multicolumn{7}{|c|}{ Rastrojo de maíz ( $\left.\mathrm{t} \mathrm{ha}^{-1}\right)$} \\
\hline 0 & $\begin{array}{l}4.4^{\mathrm{b}} \\
\pm 0.9\end{array}$ & $\begin{array}{l}3.0^{\mathrm{b}} \\
\pm 0.7\end{array}$ & $\begin{array}{l}22.6^{\mathrm{b}} \\
\pm 2.6\end{array}$ & $\begin{array}{l}29.5^{\mathrm{b}} \\
\pm 3.1\end{array}$ & $\begin{array}{l}84.4^{\mathrm{b}} \\
\pm 6.4\end{array}$ & $\begin{array}{l}107.8^{\mathrm{b}} \\
\pm 6.7\end{array}$ \\
\hline 10 & $\begin{array}{r}7.1^{\mathrm{a}} \\
\pm 0.8\end{array}$ & $\begin{array}{l}3.8^{\mathrm{a}} \\
\pm 0.8\end{array}$ & $\begin{array}{c}31.9^{\mathrm{a}} \\
\pm 3.3\end{array}$ & $\begin{array}{c}37.2^{\mathrm{a}} \\
\pm 3.4\end{array}$ & $\begin{array}{c}98.5^{\mathrm{a}} \\
\pm 5.9\end{array}$ & $\begin{array}{c}121.5^{\mathrm{a}} \\
\pm 7.4\end{array}$ \\
\hline
\end{tabular}

$\mathrm{ICC}=$ índice de contenido de clorofila; $\mathrm{BC}=$ B. curtipendula [Michx.] Torr.; $\mathrm{CG}=$ C. gayana Kunth.

${ }^{\text {ab }}$ Cifras con diferente letra dentro de una misma columna y en cada factor de variación (hidrogel y rastrojo de maíz), son diferentes $(P<0.05)$. 
Estos resultados coinciden con los reportados por algunos autores ${ }^{(22)}$, quienes indican que la adición de mantillo o cobertura vegetal, sobre una leguminosa, influyó significativamente sobre algunos atributos agronómicos como el crecimiento de las plantas, el rendimiento y la calidad nutricional del cultivo. Se ha encontrado que la aplicación de mantillo o paja en suelos salinos, aumentó significativamente el crecimiento de la planta, rendimiento de forraje en el pasto guinea ${ }^{(23)}$. Otros autores ${ }^{(24)}$, reportaron que la incorporación de mantillo al suelo para establecimiento de gramíneas, obtuvo resultados significativamente superiores en relación a tratamientos sin adición de paja, al tener mayor cantidad de vegetación de gramíneas y biomasa.

\section{- Contenido de materia seca aérea y radical de planta •}

El efecto del rastrojo estuvo relacionado con un mayor contenido de humedad edáfica y distribuida de forma más uniforme, lo cual permitió un rendimiento superior $(P \leq 0.05)$ de biomasa en $C$. gayana de $73.2 \mathrm{~g}$ planta $^{-1}$ y en $B$. curtipendula de $57.2 \mathrm{~g} \mathrm{planta}^{-1}$, respecto a no aplicar rastrojo; en promedio en cada una de las evaluaciones efectuadas, la dosis de rastrojo se asoció a rendimiento superiores de $24.9 \%$ en el pasto nativo y $25.6 \%$ en el pasto introducido, respecto del testigo (Cuadro 3). En cambio, en las dosis de hidrogel, la producción de biomasa aérea solo mostró diferencias $(P \leq 0.05)$ a los 30 DDE al aplicar $10 \mathrm{~kg}$ $\left(14.7 \mathrm{~g} \mathrm{planta}^{-1}\right)$ y $20 \mathrm{~kg}$ (13.3 $\mathrm{g}$ planta $\left.^{-1}\right)$, sin diferencia estadística en la primera dosis con el testigo (11.0 g) en B. curtipendula, En el caso de C. gayana, se obtuvieron 25.6 y $24.3 \mathrm{~g}$ en las dosis de 20 y $10 \mathrm{~kg} \mathrm{ha}^{-1}$, respectivamente, con diferencia estadística respecto al testigo (17.5 g). En las dos evaluaciones posteriores, se dejó de mostrar el efecto citado, lo cual puede estar relacionado al efecto de dilución identificado en el contenido de humedad en el suelo. La adición de rastrojo, incrementó significativamente $(P \leq 0.05)$ la biomasa de la raíz, al obtener el mayor peso al término del ciclo vegetativo en pasto nativo $(25.5 \mathrm{~g})$ e introducido (32.6 g). El incremento promedio de biomasa de la raíz en la dosis de rastrojo fue de $43.1 \%$ en nativo y $38.3 \%$ en el introducido, con respecto a no aplicar la cobertura vegetal, en las tres evaluaciones realizadas (Cuadro 4). 
Cuadro 3: Producción de biomasa aérea en peso seco de dos especies de pastos en diferentes dosis de hidrogel y rastrojo de maíz

\begin{tabular}{|c|c|c|c|c|c|c|}
\hline \multirow{3}{*}{$\begin{array}{l}\text { Dosis de retenedor de } \\
\text { humedad }\end{array}$} & \multicolumn{6}{|c|}{ Peso seco de biomasa aérea de planta $(\mathrm{g})$} \\
\hline & \multicolumn{2}{|c|}{30 DDE } & \multicolumn{2}{|c|}{$45 \mathrm{DDE}$} & \multicolumn{2}{|c|}{60 DDE } \\
\hline & $\mathbf{B C}$ & $\mathbf{C G}$ & $\mathbf{B C}$ & $\mathbf{C G}$ & $\mathbf{B C}$ & $\mathbf{C G}$ \\
\hline \multicolumn{7}{|c|}{ Hidrogel $\left(\mathrm{kg} \mathrm{ha}^{-1}\right)$} \\
\hline 0 & $\begin{array}{l}11.0^{\mathrm{b}} \\
\pm 1.7\end{array}$ & $\begin{array}{l}17.5^{\mathrm{b}} \\
\pm 3.3\end{array}$ & $\begin{array}{l}45.1^{\mathrm{a}} \\
\pm 6.0\end{array}$ & $\begin{array}{l}57.9^{\mathrm{a}} \pm \\
5.5\end{array}$ & $\begin{array}{l}53.3^{\mathrm{a}} \\
\pm 7.1\end{array}$ & $\begin{array}{l}68.0^{\mathrm{a}} \\
\pm 7.0\end{array}$ \\
\hline 10 & $\begin{array}{c}13.3 \mathrm{ab} \\
\pm 2.2\end{array}$ & $\begin{array}{l}24.3^{\mathrm{a}} \\
\pm 4.1\end{array}$ & $\begin{array}{l}42.8^{a} \\
\pm 5.9\end{array}$ & $\begin{array}{l}56.0^{\mathrm{a}} \\
\pm 5.8\end{array}$ & $\begin{array}{l}51.5^{\mathrm{a}} \\
\pm 6.3\end{array}$ & $\begin{array}{l}66.8^{\mathrm{a}} \\
\pm 7.6\end{array}$ \\
\hline 20 & $\begin{array}{l}14.7^{\mathrm{a}} \\
\pm 2.0\end{array}$ & $\begin{array}{l}25.6^{\mathrm{a}} \\
\pm 4.9\end{array}$ & $\begin{array}{l}44.7^{\mathrm{a}} \\
\pm 5.3\end{array}$ & $\begin{array}{l}56.9^{\mathrm{a}} \\
\pm 4.2\end{array}$ & $\begin{array}{l}51.6^{\mathrm{a}} \\
\pm 6.2\end{array}$ & $\begin{array}{l}67.7^{\mathrm{a}} \\
\pm 5.8\end{array}$ \\
\hline \multicolumn{7}{|c|}{ Rastrojo de maíz ( $\mathrm{t} \mathrm{ha}^{-1}$ ) } \\
\hline 0 & $\begin{array}{l}12.3^{b} \\
\pm 2.1\end{array}$ & $\begin{array}{l}20.4^{b} \\
\pm 1.6\end{array}$ & $\begin{array}{l}39.8^{b} \\
\pm 3.3\end{array}$ & $\begin{array}{l}52.3^{b} \\
\pm 2.7\end{array}$ & $\begin{array}{l}47.2^{\mathrm{b}} \\
\pm 4.4\end{array}$ & $\begin{array}{l}61.1^{\mathrm{b}} \\
\pm 2.8\end{array}$ \\
\hline 10 & $\begin{array}{l}16.1^{\mathrm{a}} \\
\pm 0.9\end{array}$ & $\begin{array}{l}28.6^{\mathrm{a}} \\
\pm 1.9\end{array}$ & $\begin{array}{l}48.8^{\mathrm{a}} \\
\pm 3.9\end{array}$ & $\begin{array}{l}61.1^{\mathrm{a}} \\
\pm 2.8\end{array}$ & $\begin{array}{l}57.2^{\mathrm{a}} \\
\pm 4.5\end{array}$ & $\begin{array}{l}73.2^{\mathrm{a}} \\
\pm 3.2\end{array}$ \\
\hline
\end{tabular}

$\mathrm{DDE}=$ días después del primer escurrimiento; $\mathrm{BC}=$ B. curtipendula [Michx.] Torr.; $\mathrm{CG}=$ C. gayana Kunth

${ }^{\text {ab }}$ Cifras con diferente letra dentro de una misma columna y en cada factor de variación (hidrogel y rastrojo de maíz), son diferentes $(P<0.05)$.

Cuadro 4: Producción de biomasa en peso seco de raíz de dos especies de pastos en diferentes dosis de hidrogel y rastrojo de maíz

\begin{tabular}{|c|c|c|c|c|c|c|}
\hline \multirow{3}{*}{$\begin{array}{l}\text { Dosis de retenedor de } \\
\text { humedad }\end{array}$} & \multicolumn{6}{|c|}{ Peso seco de biomasa radical de planta (g) } \\
\hline & \multicolumn{2}{|c|}{30 DDE } & \multicolumn{2}{|c|}{$45 \mathrm{DDE}$} & \multicolumn{2}{|c|}{60 DDE } \\
\hline & $\mathbf{B C}$ & $\mathbf{C G}$ & BC & $\mathbf{C G}$ & BC & $\mathbf{C G}$ \\
\hline \multicolumn{7}{|c|}{ Hidrogel $\left(\mathrm{kg} \mathrm{ha}^{-1}\right)$} \\
\hline 0 & $\begin{array}{l}5.0 b \\
\pm 0.5\end{array}$ & $\begin{array}{l}9.1 \mathrm{~b} \\
\pm 1.2\end{array}$ & $\begin{array}{l}19.5 \mathrm{a} \\
\pm 4.8\end{array}$ & $\begin{array}{l}24.3 \mathrm{a} \\
\pm 4.3\end{array}$ & $\begin{array}{l}22.9 \mathrm{a} \\
\pm 4.5\end{array}$ & $\begin{array}{l}29.0 \mathrm{a} \\
\pm 4.3\end{array}$ \\
\hline 10 & $\begin{array}{l}6.6 \mathrm{a} \\
\pm 1.0\end{array}$ & $\begin{array}{l}11.7 \mathrm{a} \\
\pm 2.2\end{array}$ & $\begin{array}{l}17.7 \mathrm{a} \\
\pm 3.3\end{array}$ & $\begin{array}{l}24.6 \mathrm{a} \\
\pm 3.7\end{array}$ & $\begin{array}{l}21.7 \mathrm{a} \\
\pm 3.2\end{array}$ & $\begin{array}{l}29.5 \mathrm{a} \\
\pm 4.1\end{array}$ \\
\hline 20 & $\begin{array}{r}6.3 \mathrm{a} \\
\pm 0.8\end{array}$ & $\begin{array}{l}11.9 \mathrm{a} \\
\pm 2.1\end{array}$ & $\begin{array}{l}19.0 \mathrm{a} \\
\pm 3.5\end{array}$ & $\begin{array}{l}25.5 \mathrm{a} \\
\pm 3.7\end{array}$ & $\begin{array}{l}22.5 \mathrm{a} \\
\pm 3.6\end{array}$ & $\begin{array}{l}28.9 \mathrm{a} \\
\pm 3.6\end{array}$ \\
\hline \multicolumn{7}{|c|}{ Rastrojo de maíz ( $\left.\mathrm{t} \mathrm{ha}^{-1}\right)$} \\
\hline 0 & $\begin{array}{l}4.7 \mathrm{~b} \\
\pm 1.8\end{array}$ & $\begin{array}{l}9.2 \mathrm{~b} \\
\pm 1.1\end{array}$ & $\begin{array}{l}15.5 b \\
\pm 2.4\end{array}$ & $\begin{array}{l}20.9 b \\
\pm 1.5\end{array}$ & $\begin{array}{l}19.4 b \\
\pm 1.9\end{array}$ & $\begin{array}{l}25.3 \mathrm{~b} \\
\pm 1.3\end{array}$ \\
\hline 10 & $\begin{array}{r}7.3 \mathrm{a} \\
\pm 0.6\end{array}$ & $\begin{array}{l}13.9 a \\
\pm 1.4\end{array}$ & $\begin{array}{l}22.1 \mathrm{a} \\
\pm 2.2\end{array}$ & $\begin{array}{l}28.2 \mathrm{a} \\
\pm 1.2\end{array}$ & $\begin{array}{l}25.5 \mathrm{a} \\
\pm 2.3\end{array}$ & $\begin{array}{l}32.6 \mathrm{a} \\
\pm 1.6\end{array}$ \\
\hline
\end{tabular}

$\mathrm{DDE}=$ días después del primer escurrimiento; $\mathrm{BC}=$ B. curtipendula [Michx.] Torr.; $\mathrm{CG}=$ C. gayana Kunth.

${ }^{a b}$ Cifras con diferente letra dentro de una misma columna y en cada factor de variación (hidrogel y rastrojo de maíz), son diferentes $(P<0.05)$. 
Estos resultados coinciden con estudios que identificaron una mayor cantidad de biomasa al aplicar mantillo o rastrojo sobre la superficie del suelo ${ }^{(24)}$. Otros autores ${ }^{(23)}$, obtuvieron aumentos significativamente mayores en el rendimiento de forraje en pasto guinea al adicionar paja al suelo. El hidrogel, ha mostrado un uso más eficiente del agua, lo cual mejora el crecimiento de plantas ${ }^{(25)}$; sin embargo, este efecto no fue sostenido en el tiempo de evaluación. Respecto a los pastos introducidos, donde se indica que ofrecen ventajas a las variedades nativas, tales como su rápida adaptación y crecimiento $^{(26)}$, se señala que la principal ventaja de estos pastos es la producción de biomasa, la cual es superior en comparación a algunas especies nativas ${ }^{(27)}$, lo cual coincide con este estudio.

\section{" Conclusiones e implicaciones V}

El pasto nativo (B. curtipendula) tiene mejores ventajas con respecto a la variedad introducida ( $C$. gayana) en fases fenológicas iniciales, mediante una mayor capacidad germinativa y mayor velocidad de germinación; sin embargo, el pasto introducido registró una mayor altura, mejor amacollamiento y un mayor índice de clorofila, y como consecuencia una mayor producción de biomasa. La aplicación de rastrojo incrementó en un $16.9 \%$ el contenido de humedad del suelo, lo cual incrementó significativamente la producción de biomasa aérea. Las dosis de hidrogel sólo influyeron en un mejor porcentaje de establecimiento y un mayor contenido de humedad edáfica durante los primeros 15 días de realizado el trasplante, sin ningún efecto posterior durante la fase de crecimiento y desarrollo de los pastos.

\section{Agradecimientos}

Al personal técnico y científico del Centro Nacional de Investigación Disciplinaria en Relaciones Agua Suelo Planta Atmósfera del INIFAP en Gómez Palacio, Dgo. por el apoyo técnico. 


\section{- Literatura citada:}

1. Velásquez VMA, Alba AA, Gutiérrez LR, García EG. Prácticas de restauración de suelos para la conservación del agua. Instituto Nacional de Investigaciones Forestales, Agrícolas y Pecuarias. CIRNOC. Zacatecas. Folleto Técnico No. 46. 2012.

2. Asner PG, Elmore JA, Olander PL, Martin ER, Harris TA. Grazing systems, ecosystem responses, and global change. Annu Rev Environ Resour 2004;29:261-299.

3. Cornelis W M. Hydroclimatology of wind erosion in arid and semiarid environments. In: Dryland ecohydrology. D’Odorico, Porporato A, editors. Netherlands: Springer; 2006:141-161.

4. Kéfi S, Rietkerk M, Alados LC, Pueyo Y, Papanastasis PV, ElAich A, Ruiter PC. Spatial vegetation patterns and imminent desertification in Mediterranean arid ecosystems. Nature 2007;449(7159):213-217.

5. Pinedo AC, Hernández QNS, Melgoza CA, Rentería VM, Vélez SVC, Morales NC, et al. Diagnóstico actual y sustentabilidad de los pastizales del estado de Chihuahua ante el cambio climático. Cuerpo Académico de Recursos Naturales y Ecología (UACHCA16). Facultad de Zootecnia y Ecología, Universidad Autónoma de Chihuahua. Chihuahua, México. 2003.

6. Chávez RE, González CG, González BJL, López DA. La evapotranspiración en la cuenca baja y media del río Nazas. En: Oswald SU. Retos de la investigación del agua en México. Cuernavaca, Mor. México. UNAM, Centro Regional de Investigaciones Multidisciplinarias. 2011.

7. Cruz MA, Pedroza SA, Trejo CR, Sánchez CI, Samaniego GJA, Hernández SJR. Captación de agua de lluvia y retención de humedad edáfica en el establecimiento de buffel (Cenchrus ciliaris L.). Téc Pecu Mex 2016;7(2):159-172.

8. IMTA (Instituto Mexicano de Tecnología del Agua). Extractor rápido de información climatológica (ERIC III). 2.0. México, D.F. Servicio Meteorológico Nacional. 2009.

9. SNF Inc. Water retainers for soils and substrates. http://snf.com.au/downloads/ Aquasorb_E.pdf Consultado 10 Nov, 2016.

10. González DJR, Gómez MS, López DA. El trasplante garantiza establecer zacates forrajeros en suelos salinos y arcillosos [resumen]. Congreso Internacional de Manejo de Pastizales. Durango. Dgo. 2015:464. 
11. SAS. Institute Statistical Analysis System. The SAS System for Windows Version 9.3. North Carolina, USA: SAS Institute Inc. Cary; 1976.

12. Carrillo SSM, Arredondo MT, Huber-Sannwald E, Flores RJ. Comparación en la germinación de semillas y crecimiento de plántulas entre gramíneas nativas y exóticas del pastizal semiárido. Tec Pecu Mex 2009;47(3):299-312.

13. Esqueda CMH, Melgoza CA, Sosa CM, Carrillo RR, Jiménez CJ. (2012). Emergencia y sobrevivencia de gramíneas con diferentes secuencias de humedad-sequía en tres tipos de suelo. Tec Pecu Mex 2012;43(1):101-115.

14. Barón CA, Barrera RIX, Boada ELF, Rodríguez NG. Evaluación de hidrogeles para aplicaciones agroforestales. Rev Ing Invest 2007;27(3):35-44.

15. Dorraji SS, Golchin A, Ahmadi S. The effects of hydrophilic polymer and soil salinity on corn growth in sandy and loamy soils. Clean-Soil, Air, Water 2010;38(7):584-591.

16. Narjary B, Aggarwal P, Singh A, Chakraborty D, Singh R. Water availability in different soils in relation to hydrogel application. Geoderma 2012;(187-188):94-101.

17. Obalum SE, Igwe CA, Eze OM. Soil moisture dynamics under rainfed sorghum and soybean on contrasting tillage-mulch seedbeds in a mineral sandy loam at derived savanna of south-eastern Nigeria. Arch Agron Soil Sci 2012;58(11):1205-1227.

18. Taparauskienė L, Miseckaitė O. Effect of mulch on soil moisture depletion and strawberry yield in Sub-Humid area. Pol J Environ Stud 2014;23(2):475-482.

19. Zhao Y, Pang H, Wang J, Huo L, Li Y. Effects of straw mulch and buried straw on soil moisture and salinity in relation to sunflower growth and yield. Field Crop Res 2014;161:16-25.

20. Nissen J, Ovando C. Efecto de un hidrogel humectado aplicado a las raíces de Nothofagus obliqua (MIRB.) OERST. y Nothofagm dombeyi (MIRB.) OERST. durante su trasplante. Agro Sur 1999;27(2):48-58.

21. Julca OA, Meneses FL, Blas SR, Bello AS. La materia orgánica, importancia y experiencia de su uso en la agricultura. Idesia (Arica) 2006;24(1):49-61.

22. Ravisankar N, Balakrishnan M, Ambast SK, Srivastava RC, Bommayasamy N, Subramani $\mathrm{T}$. Influence of irrigation and crop residue mulching on yield and water productivity of table purpose groundnut (Arachis hypogaea) in humid tropical island. Legume Res: An Intern J 2014;37(2):195-200. 
23. Kusmiyati F, Sumarsono S, Karno K, Pangestu E. Effect of mulch and mixed cropping grass-legume at saline soil on growth, forage yield and nutritional quality of guinea grass. J Indonesian Trop Anim Agric 2013;38(1):72-78.

24. Beggy HM, Fehmi JS. Effect of surface roughness and mulch on semi-arid revegetation success, soil chemistry and soil movement. Catena 2016;143:215-220.

25. El-Hady OA, Safia MA, Abdel KAA. Sand-Compost-Hydrogel mix for low cost production of tomato seedlings. Egypt J Soil Sci 2002;42:767-782.

26. Velázquez MM, Hernández GFJ, Cervantes BJF, Gámez VHG. Establecimiento de pastos nativos e introducidos en zonas áridas de México. San Luis Potosí, SLP México. Instituto Nacional de Investigaciones Forestales, Agrícolas y Pecuarias. CIR-Noreste. 2015.

27. Loredo OC, Beltrán LS. Establecimiento y manejo de pasto Klein en el altiplano potosino. San Luis Potosí, SLP México. Instituto Nacional de Investigaciones Forestales, Agrícolas y Pecuarias. CIR-Noreste. Folleto técnico No. 24. 2001. 